Article

\title{
State of Charge Estimation of Lithium-Ion Batteries Based on Fuzzy Fractional-Order Unscented Kalman Filter
}

\author{
Liping Chen ${ }^{1, * \mathbb{D}}$, Yu Chen ${ }^{1}$, António M. Lopes ${ }^{2}{ }^{\mathbb{D}}$, Huifang Kong ${ }^{1}$ and Ranchao $\mathrm{Wu}^{3}$ \\ 1 School of Electrical Engineering and Automation, Hefei University of Technology, Hefei 230009, China; \\ 2019170408mail@hfut.edu.cn (Y.C.); 1989800024@hfut.edu.cn (H.K.) \\ 2 LAETA/INEGI, Faculty of Engineering, University of Porto, Rua Dr. Roberto Frias, 4200-465 Porto, Portugal; \\ aml@fe.up.pt \\ 3 School of Mathematics, Anhui University, Hefei 230039, China; rcwu@ahu.edu.cn \\ * Correspondence: lip_chen@hfut.edu.cn
}

Citation: Chen, L.; Chen, Y.;

Lopes, A.M.; Kong, H.; Wu, R. State of Charge Estimation of Lithium-Ion Batteries Based on Fuzzy Fractional-Order Unscented Kalman Filter. Fractal Fract. 2021, 5, 91. https://doi.org/10.3390/ fractalfract5030091

Academic Editor: Ivanka Stamova

Received: 25 June 2021

Accepted: 6 August 2021

Published: 8 August 2021

Publisher's Note: MDPI stays neutral with regard to jurisdictional claims in published maps and institutional affiliations.

Copyright: (c) 2021 by the authors. Licensee MDPI, Basel, Switzerland. This article is an open access article distributed under the terms and conditions of the Creative Commons Attribution (CC BY) license (https:/ / creativecommons.org/licenses/by/ $4.0 /)$.

\begin{abstract}
The covariance matrix of measurement noise is fixed in the Kalman filter algorithm. However, in the process of battery operation, the measurement noise is affected by different charging and discharging conditions and the external environment. Consequently, obtaining the noise statistical characteristics is difficult, which affects the accuracy of the Kalman filter algorithm. In order to improve the estimation accuracy of the state of charge (SOC) of lithium-ion batteries under actual working conditions, a fuzzy fractional-order unscented Kalman filter (FFUKF) is proposed. The algorithm combines fuzzy inference with fractional-order unscented Kalman filter (FUKF) to infer the measurement noise in real time and take advantage of fractional calculus in describing the dynamic behavior of the lithium batteries. The accuracy of the SOC estimation under different working conditions at three different temperatures is verified. The results show that the accuracy of the proposed algorithm is superior to those of the FUKF and extended Kalman filter (EKF) algorithms.
\end{abstract}

Keywords: Kalman filter; state of charge; fuzzy inference; lithium-ion batteries

\section{Introduction}

The automobile industry has payed extensive attention to new energies to reduce the emissions of greenhouse gases [1]. An important component of the new energy vehicles is the power battery system. Lithium-ion batteries have the advantage of high energy density and excellent performance cycles [2]. However, their safe and effective management are crucial. The battery management system (BMS) is critical and the state of charge (SOC) estimation plays a vital role in the BMS $[3,4]$. However, the SOC of the power battery cannot be measured directly, and some efficient and accurate estimation methods must be employed. Compared with electrochemical and data-driven models, the equivalent circuit model (ECM) was widely adopted in recent years, which uses ideal resistors, capacitors, constant voltage sources and other circuit devices to form a circuit network that describes the characteristics of power batteries [5]. In addition, to obtain a reliable battery model, the SOC estimation also requires a high precision algorithm. Recently, effective estimation methods have been presented, such as the open circuit voltage (OCV) method [6], amperehour integration method [7,8], Kalman filter algorithm, neural network method [9,10], sliding mode observer [11,12], $H_{\infty}$ filter [13,14], adaptive particle filter [15] and others. Each of these methods presents advantages and disadvantages. For example, the OCV is the most direct method, but it requires the battery to stand for a long enough time. The ampere-hour integration method is classic, easy and widely used, but its initial SOC is difficult to obtain. The neural network method is popular with high estimation accuracy, but it requires a large amount of experimental data as prior knowledge, and these data can fully reflect the characteristics of the battery. 
The Kalman filter is currently the most used estimation algorithm. It includes the extended Kalman filter (EKF) [16-18], unscented Kalman filter (UKF) [19,20], adaptive Kalman filter [21-23], fuzzy unscented Kalman filter [24] and other variants. The EKF uses the Taylor expansion to linearize high-order terms, resulting in error accumulation during the iterative process. The UKF uses the unscented transformation to linearize the nonlinear function of random variables by linear regression. Generally speaking, the unscented transformation is more accurate than the Taylor series approach. However, the statistical characteristics of the measurement noise are vital for the UKF, being difficult to obtain accurately. Indeed, the statistical characteristics of the noise are affected by uncertain factors, such as the system noise, which causes the UKF to converge slowly and even to diverge. The adaptive Kalman filter method can estimate the process and the observation noise online, improving the accuracy of the estimation [25]. However, when the nonlinearity is strong, the estimation accuracy is limited.

Recently, it was found that fractional-order ECM (FECM), where constant phase elements (CPE) are used instead of ideal capacitors [26], have advantages for describing the dynamic behavior of lithium batteries. The FECM can accurately simulate the doublelayer effect of the battery electrode. Therefore, SOC algorithms based on FECM, such as fractional-order unscented Kalman filter (FUKF) [27,28] and fractional-order extended Kalman filter (FEKF) [29-31] have been proposed. Experimental results have also shown that these methods improve the accuracy of the estimation when compared with the ECM. However, the statistical characteristics of the measurement noise are still hard to obtain accurately [32] and affect greatly the accuracy of the SOC estimation. In order to mitigate this shortcoming, a new fuzzy fractional-order unscented Kalman filter (FFUKF) that combines fuzzy inference and FUKF is proposed. This method can infer the measurement noise in real time and has higher accuracy compared with traditional algorithms, according to the difference between the actual and the theoretical value of the noise measurements. The covariance matrix of the measurement noise is adjusted continuously to make the FUKF more adaptive and accurate.

The main objective of this paper is (1) to propose a fractional-order second-order RC equivalent circuit model of lithium batteries based on particle swarm optimization (PSO), (2) to derive a FFUKF to solve the influence of measurement noise on SOC estimation accuracy, (3) to test the FFUKF under different working conditions and compare its performance with the FUKF and EKF.

The paper is organized as follows. Section 2 introduces the fractional-order model and its parameter identification. Section 3 presents the fuzzy controller. Section 4 lists the steps of the FFUKF algorithm. Section 5 compares the results with those obtained with existing algorithms. Section 6 outlines the main conclusions.

\section{Theory and Method Research}

\subsection{Fractional-Order Calculus}

In contrast with the integer-order derivative, the fractional-order derivative have many definitions, such as the Grünwald-Letnikov (GL), Riemann-Liouville (RL), and Caputo formulations. Here, the GL definition is used [33]:

$$
\begin{gathered}
t_{0} D_{t}^{\alpha} x(t)=\lim _{\Delta T \rightarrow 0}\left(\frac{1}{\Delta T^{\alpha}}\right) \sum_{j=0}^{[t / \Delta T]}(-1)^{j}\left(\begin{array}{c}
\alpha \\
j
\end{array}\right) x(t-j \Delta T), \\
\left(\begin{array}{c}
\alpha \\
j
\end{array}\right)=\frac{\Gamma(\alpha+1)}{\Gamma(j+1) \cdot \Gamma(\alpha-j+1)} .
\end{gathered}
$$

with $\Gamma(\alpha)$ given by:

$$
\Gamma(\alpha)=\int_{0}^{+\infty} \xi^{\alpha-1} e^{-\xi} d \xi
$$


where $t$ denotes the variable, with lower bound $t_{0}, \Delta T$ stands for the sampling time, and $[t / \Delta T]$ is the memory length. The continuous-time GL derivative $t_{0} D_{t}^{\alpha}$ can be discretized with a fixed memory length $L$, yielding:

$$
D^{\alpha} x_{k+1}=\frac{1}{\Delta T^{\alpha}} x_{k+1}+\left(\frac{1}{\Delta T^{\alpha}}\right) \sum_{j=1}^{L+1}(-1)^{j}\left(\begin{array}{c}
\alpha \\
j
\end{array}\right) x_{k+1-j} .
$$

\subsection{Fractional-Order Model}

In order to describe accurately the internal electrochemical reaction that occurs in a battery, and to design an accurate and reliable lithium-ion battery SOC estimation algorithm, an accurate model is necessary. It has been shown that the ECM describes well the battery characteristics and that a second-order RC model yields good results in terms of accuracy and computational complexity [34]. Usually, the ECM includes two ideal resistors. However, for the complex electrochemical reactions inside the battery, the ideal capacitance cannot be simulated reasonably. Therefore, the CPE has been used instead of ideal capacitors, which resulted in the FECM. The impedance of a CPE is given by:

$$
Z(s)=\frac{1}{C s^{\alpha}} .
$$

The fractional-order RC circuit used here is shown in Figure 1, where $U_{o c}$ stands for the open circuit voltage, $R_{0}$ is the ohmic internal resistance, and $R_{1}$ and $R_{2}$ are the electrochemical polarization and concentration polarization resistances, respectively. The $C P E_{1}$ and $C P E_{2}$ stand for the fractional capacitors, $V_{0}$ represents the terminal voltage of the battery, and $I$ corresponds to the load current. If we denote by $V_{1}$ and $V_{2}$ the voltages on the two parallel associations, respectively, then the dynamic equations can be expressed as:

$$
\left\{\begin{array}{l}
D^{\alpha} V_{1}(t)=-\frac{V_{1}(t)}{R_{1} C_{1}}+\frac{I(t)}{C_{1}} \\
D^{\beta} V_{2}(t)=-\frac{V_{2}(t)}{R_{2} C_{2}}+\frac{I(t)}{C_{2}} .
\end{array}\right.
$$

where $\alpha, \beta \in(0,1)$ are the fractional orders of $C P E_{1}$ and $C P E_{2}$, respectively. The variable $Q_{n}$ is the nominal capacity of the lithium-ion battery and $\eta$ is the Coulomb efficiency. The SOC of the lithium battery can be written as:

$$
\frac{d S O C(t)}{d t}=-\frac{\eta}{Q_{n}} I(t) .
$$

It follows from the Kirchhoff's voltage law that the output equation is given by:

$$
V_{0}(t)=U_{o c}-I(t) R_{0}-V_{1}(t)-V_{2}(t),
$$

where OCV is a nonlinear function of the SOC. Equation (8) has been used to describe the OCV-SOC relationship $[35,36]$, which is usually expressed as:

$$
f[\theta(t)]=U_{o c}=\sum_{i=0}^{4} a_{i} S O C(t)
$$

where $a_{i}(i=0, \cdots, 4)$ are polynomial coefficients.

Further, the state space equation of the lithium-ion battery can be established as:

$$
\left\{\begin{aligned}
D^{\eta} x(t) & =A x(t)+B u(t) \\
y(t) & =C x(t)+D u(t)
\end{aligned}\right.
$$


where $\eta=[\alpha, \beta, 1]^{T}$ represents the incommensurate order vector, $x(t)=\left[V_{1}, V_{2}, S O C\right]^{T}$ is the state vector, $u(t)$ denotes the system input (battery current $I(t))$ and $y(t)$ represents the system output (battery terminal voltage $V_{0}$ ). The matrices $A, B, C$ and $D$ are given as:

$$
\begin{aligned}
& A=\left[\begin{array}{ccc}
-\frac{1}{R_{1} C_{1}} & 0 & 0 \\
0 & -\frac{1}{R_{2} C_{2}} & 0 \\
0 & 0 & 0
\end{array}\right], B=\left[\begin{array}{c}
\frac{1}{C_{1}} \\
\frac{1}{C_{2}} \\
-\frac{\eta}{Q_{n}}
\end{array}\right], \\
& C=\left[\begin{array}{lll}
-1 & -1 & \frac{f[\theta(t)}{S O C}(t)
\end{array}\right], D=-R_{0} .
\end{aligned}
$$

With Equation (4) in mind, model (10) can be written in discrete time:

$$
\left\{\begin{array}{c}
x_{k+1}=A_{1} x_{k}+B_{1} u_{k}-\sum_{j=2}^{L+1}(-1)^{j} \gamma_{j}^{\eta} x_{k+1-j}, \\
y_{k}=f\left(\theta_{k}\right)-V_{1 k}-V_{2 k}-R_{0} I_{k} .
\end{array}\right.
$$

The matrices of $A_{1}, B_{1}$ and $\gamma_{j}^{\eta}$ are as follows:

$$
\begin{gathered}
A_{1}=\operatorname{diag}\left((\Delta T)^{\alpha},(\Delta T)^{\beta},(\Delta T)\right) A+\operatorname{diag}(\alpha, \beta, 1), \\
B_{1}=\operatorname{diag}\left((\Delta T)^{\alpha},(\Delta T)^{\beta},(\Delta T)\right) B, \\
\gamma_{j}^{\eta}=\operatorname{diag}\left(\left(\begin{array}{c}
\alpha \\
j
\end{array}\right),\left(\begin{array}{c}
\beta \\
j
\end{array}\right),\left(\begin{array}{c}
1 \\
j
\end{array}\right)\right) .
\end{gathered}
$$

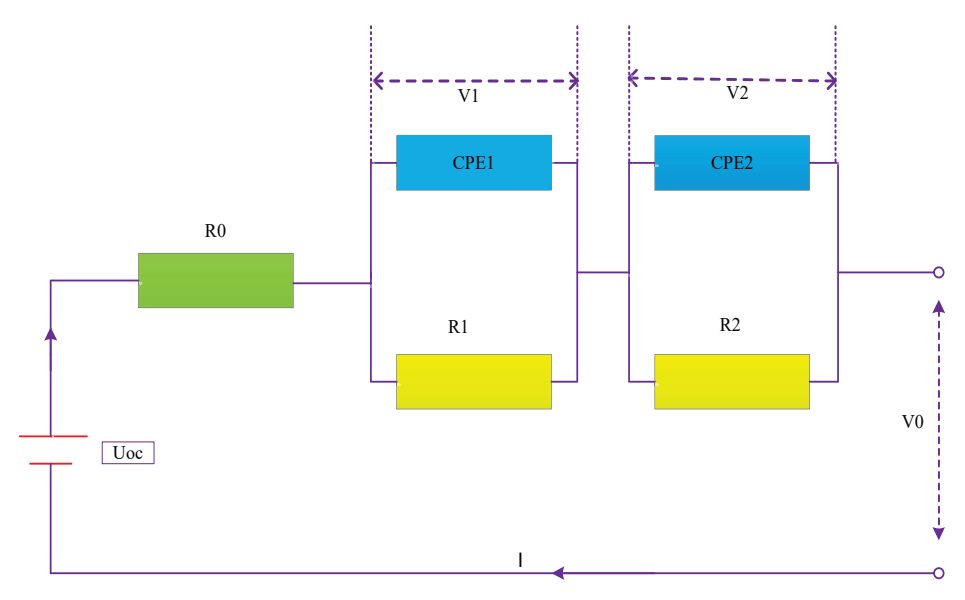

Figure 1. Equivalent circuit model of a lithium battery.

\subsection{Model Parameter Identification and Validation}

The main current and voltage data of the battery INR 18650-20R with a capacity $2000 \mathrm{mAH}$ are provided by the CALCE Battery Research Group. The experimental platform is composed by the test samples, a thermal chamber (Weiss-Voetsch, Germany), an Arbin BT2000 battery test system (ARBIN, TX, USA), and a PC with Arbin software (V4.27, Caltest Instruments Ltd, Petersfield, UK) to give orders to the test system and monitor data information. All tests were performed for 0.8 and 0.5 battery level at $0{ }^{\circ} \mathrm{C}, 25^{\circ} \mathrm{C}$ and $45^{\circ} \mathrm{C}$. We use three typical current and voltage test data sets of the vehicle operating conditions: Dynamic Stress Test (DST), Federal Urban Driving Schedule (FUDS) and Beijing Dynamic Stress Test (BJDST). Through the analysis of the established fractional-order model, we 
need to identify twelve parameters, which are $a_{0}, a_{1}, a_{2}, a_{3}, a_{4}, R_{0}, R_{1}, C_{1}, R_{2}, C_{2}, \alpha$ and $\beta$. Here, a PSO is used as the identification algorithm, but other methods are possible to estimate the parameters, such as, for example, the observer method $[37,38]$. The PSO originated in the study of the behavior of birds. The basic idea of the algorithm is to find optimal solutions through collaboration and information sharing between individuals in a group. The advantage of PSO is that it is simple and easy to implement with a limited number of parameter adjustments. Here, we set the goal of minimizing the root mean square error (RMSE) between the measured and the estimated voltages. Therefore, we define the objective function $E$ as:

$$
\min E=\sum_{k=1}^{n}\left[V_{o}(k)-\widehat{V}_{o}(k)\right]^{2},
$$

where $V_{o}(k)$ and $\widehat{V}_{o}(k)$ are the measured and estimated voltages, respectively, and $n$ is the number of the sampling points.

Table 1 shows the results of the parameter identification of the fractional-order model. For model validation, the DST is used. The current and voltage profiles of the DST at a temperature of $25^{\circ} \mathrm{C}$ are shown in Figure 2. We verify that the DST condition is composed of many small cycles, each with a duration of $350 \mathrm{~s}$ [39]. Here, to reduce the complexity, a cycle is selected for parameter identification. Figure 3 presents the current and voltage profiles of a cycle. Figures 4 and 5 show the accuracy of the fractional-order model, which is also compared with an integer-order model. From Figure 5, we observe that the error of the fractional-order model can be kept within $40 \mathrm{mV}$. However, the maximum error of the integer-order model is $80 \mathrm{mV}$. The RMSE of the two models is 0.0125 and 0.0573 , respectively. Therefore, from Figures 3-5, one can see that the fractional-order model can perform better than the integer-order one in modeling the change of terminal voltage, and that the fractional-order model is more accurate.

Table 1. The results of the fractional-model parameter identification.

\begin{tabular}{cccccc}
\hline$a_{0}$ & $a_{1}$ & $a_{2}$ & $a_{3}$ & $a_{4}$ & $R_{0}$ \\
\hline 2.4877 & 1.8243 & 0.6608 & 1.1131 & -3.2348 & 0.0687 \\
\hline$R_{1}$ & $C_{1}$ & $R_{2}$ & $C_{2}$ & $\alpha$ & $\beta$ \\
\hline 0.5975 & 264.25 & 1.2679 & 448.54 & 0.4325 & 0.4380 \\
\hline
\end{tabular}

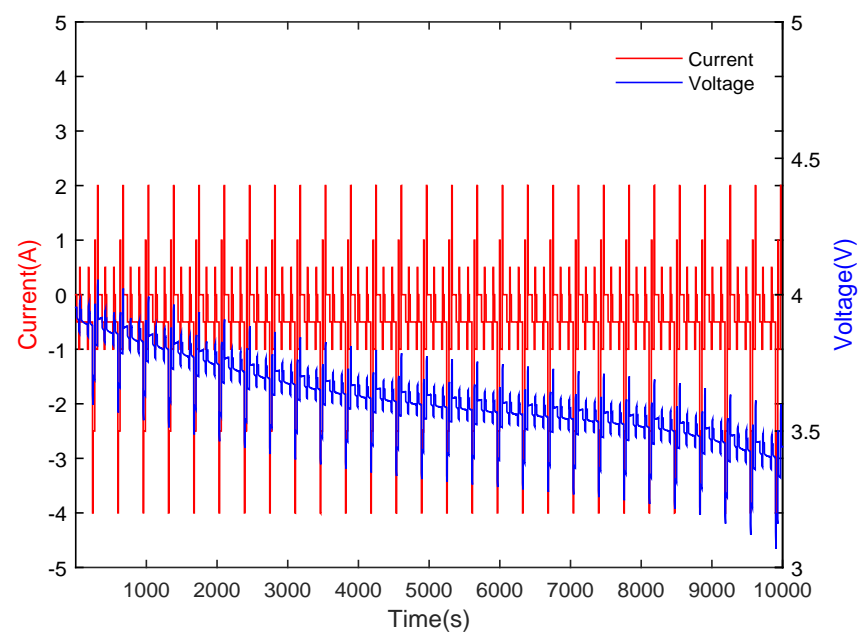

Figure 2. Current and voltage profiles of the operation conditions: DST. 


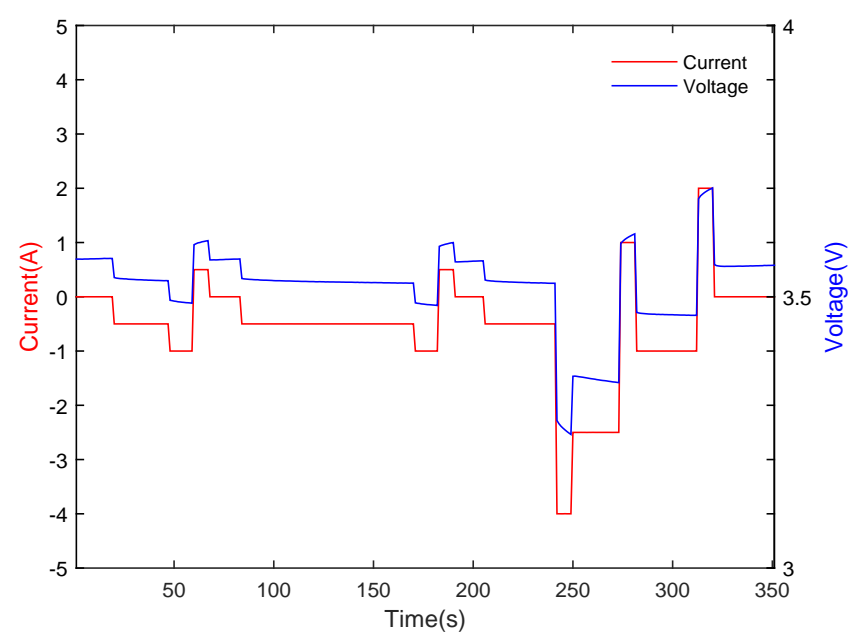

Figure 3. Current and voltage data of a DST cycle.

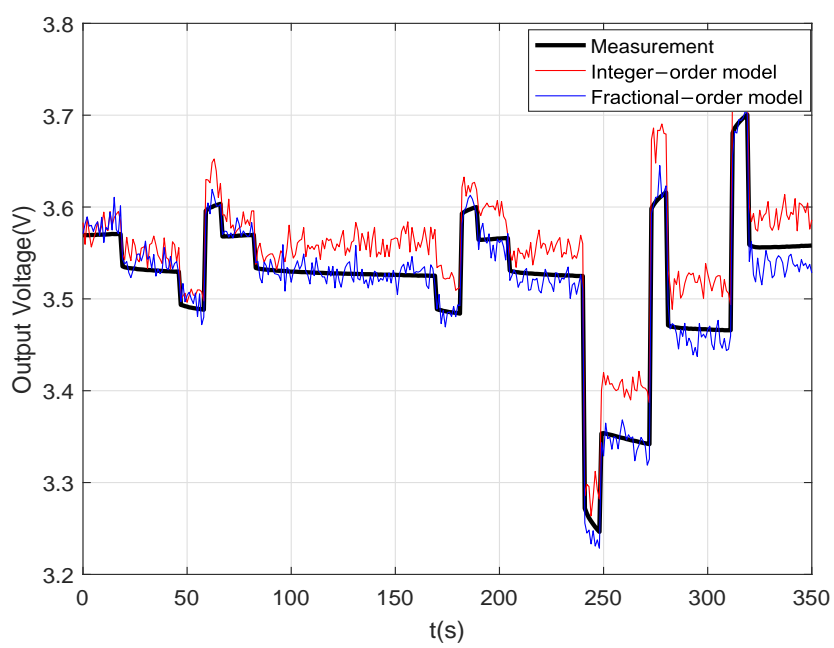

Figure 4. Accuracy verification of the model.

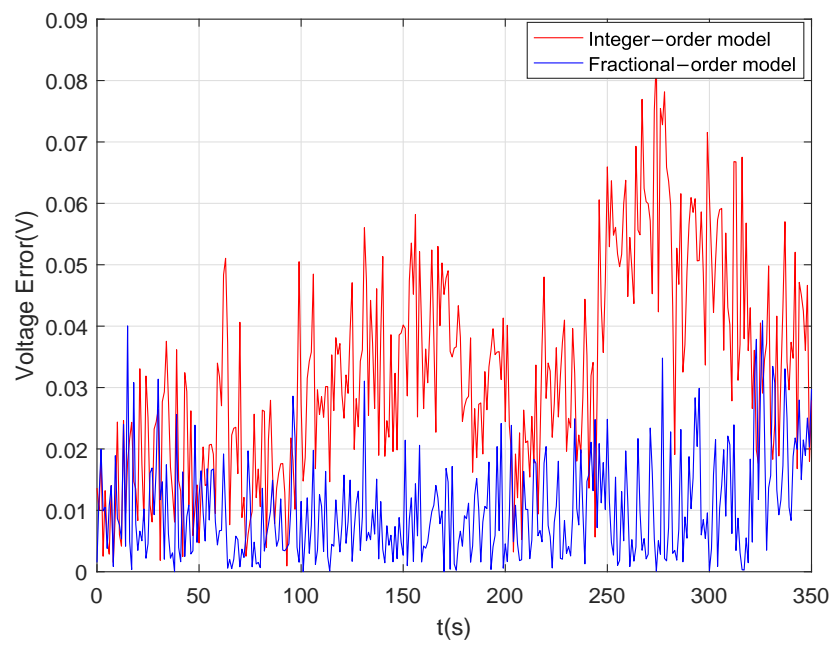

Figure 5. Output terminal voltage error curve.

\section{Fuzzy Controller}

The selection of the membership functions is very significant for the performance of a fuzzy controller. There is no ready-made rule for the establishment of the membership 
functions, and most of the methods are still based on experience and experimentation. The membership functions used here are shown in the Figure 6.

Fuzzy control is an effective method to solve the influence of measurement noise on the accuracy of SOC estimation in a complex environment. The fuzzy controller includes three main parts, as illustrated in Figure 7. First, we start the fuzzy processing on the input value $G_{k}$, based on the input membership function, to obtain the corresponding fuzzy index, where $G_{k}$ is the difference between the theoretical and actual covariances $M_{k}$ and $N_{k}$. Second, we establish the fuzzy rules as shown in Table 2. Large observation noise leads to changes in the actual covariance $N_{k}$, while the theoretical covariance $M_{k}$ is affected by changes in the observation noise variance $V_{k}$. To maintain the consistency regarding changes between $M_{k}$ and $N_{k}$, when the observation noise is large (small), we adjust the output value $\mu_{k}$ to expand (reduce) $V_{k}$ so that $G_{k}$ is close to 0 . Finally, we perform the inverse fuzzy processing, according to the output membership function, to obtain $\mu_{k}$. Therefore, we can get a new noise variance $\widehat{V_{k}}$ to perform the update of the observation noise variance adaptively.
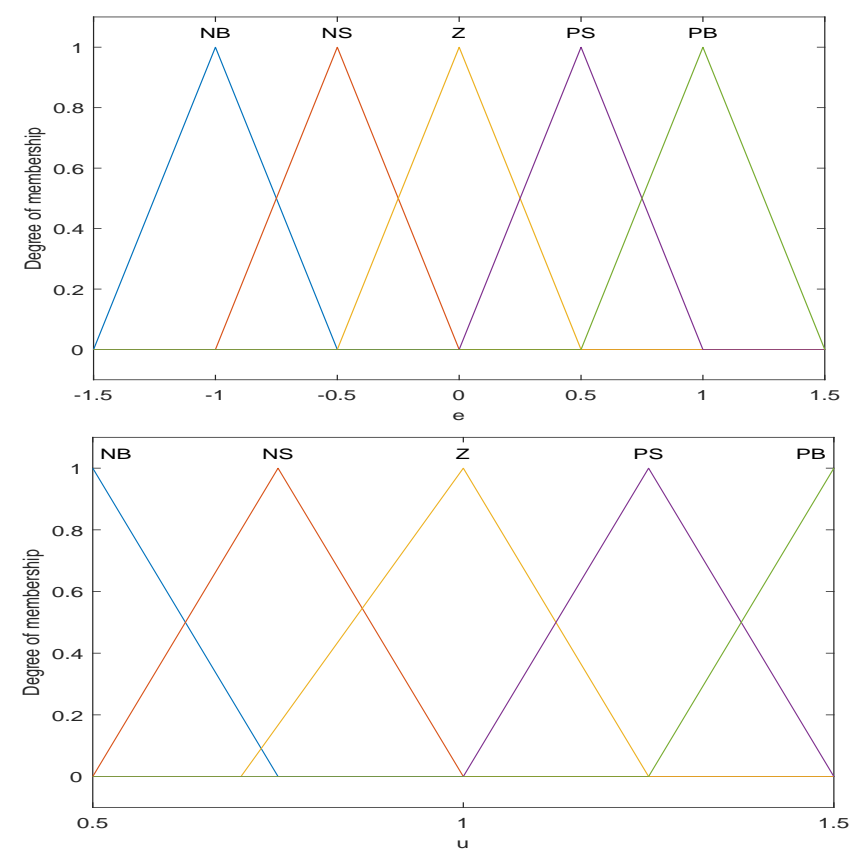

Figure 6. Input and output membership functions.

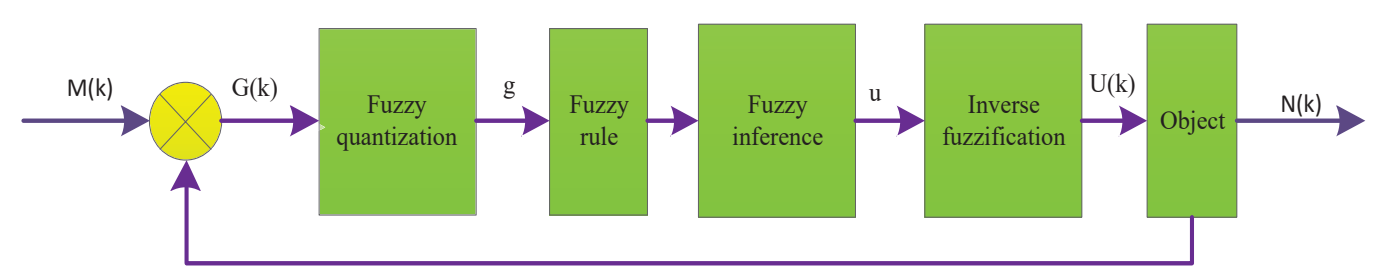

Figure 7. Fuzzy controller diagram.

Table 2. Fuzzy rules.

\begin{tabular}{clllll}
\hline Input fuzziness & NB & NS & Z & PS & PB \\
\hline Output fuzziness & NB & NS & Z & PS & PB \\
\hline
\end{tabular}

\section{SOC Estimation}

Firstly, the observability of the battery model is analyzed. A method to determine the observability of continuous time multi-order fractional-order systems was proposed in [40]. 
The fractional-order system is observable if its observability matrix is full rank. According to the Equations (10)-(12), we can get that the observability matrix $O$ of the system is:

$$
O=\left[\begin{array}{ccc}
1 & 1 & -\frac{U_{o c}}{S O C} \\
-\frac{1}{R_{1} C_{1}} & 0 & 0 \\
0 & -\frac{1}{R_{2} C_{2}} & 0
\end{array}\right]
$$

It is easy to see that this matrix is full rank. Therefore, the second-order FECM is observable. Compared to the FUKF, the FFUKF reveals higher accuracy and efficiency for SOC estimation. In this section, we discuss in detail the main steps of the FFUKF algorithm. The fractional-order system is given by:

$$
\left\{\begin{aligned}
D^{\eta} x_{k+1} & =f\left(x_{k}, u_{k}\right)+\omega_{k \prime} \\
x_{k+1} & =D^{\eta} x_{k+1}-\sum_{j=1}^{L+1}(-1)^{j} \gamma_{j} x_{k+1-j} \\
y_{k} & =h\left(x_{k}\right)+V_{k}
\end{aligned}\right.
$$

where $x_{k}$ represents the system state variable, $u_{k}$ and $y_{k}$ denote the system input and output, respectively, $f\left(x_{k}, u_{k}\right)$ stands for the system process model, and $h\left(x_{k}\right)$ is the system measurement model. The symbol $\omega_{k}$ represents a Gaussian process noise and $V_{k}$ corresponds to measurement noise. The variables $Q$ and $R$ represent the covariance matrices of $\omega_{k}$ and $V_{k}$, respectively.

The flow chart of the FFUKF is illustrated in Figure 8. The detailed FFUKF steps are presented as follows:

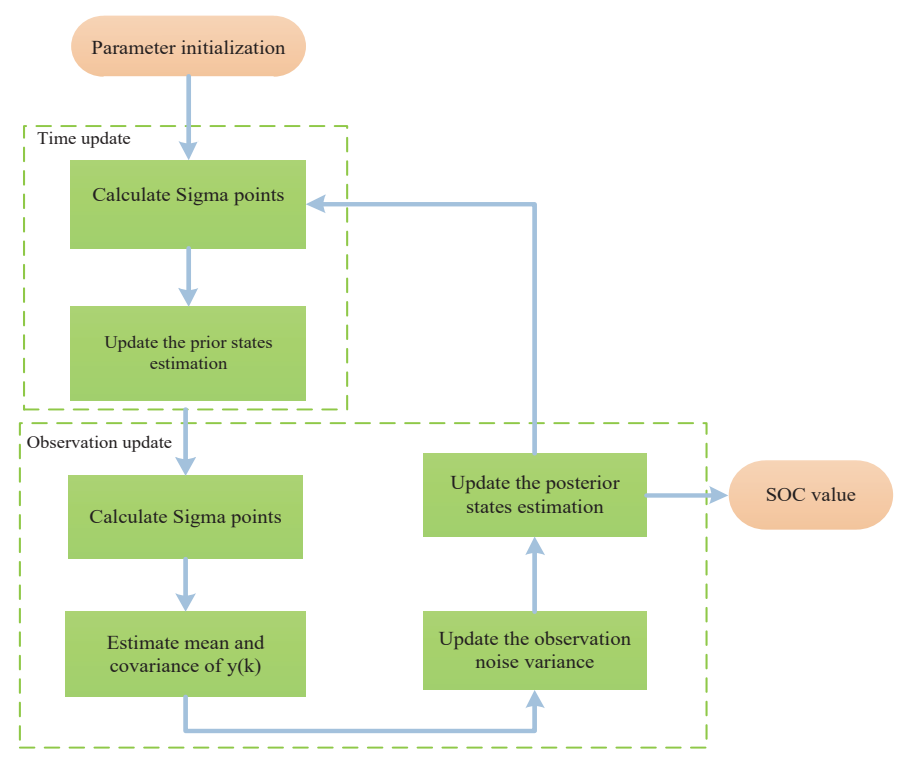

Figure 8. Flow chart of the FFUKF.

\section{Initialization}

(1) Give the initial state $x_{0}, Q, R$ and state error covariance $P$.

2 Time updating

(1) Calculate sigma points using the singular value decomposition: 


$$
\left\{\begin{aligned}
P_{k-1 \mid k-1} & =U_{k-1} S_{k-1} V_{k-1}^{T} \\
x_{0, k-1 \mid k-1} & =\widehat{x}_{k-1 \mid k-1} \\
x_{i, k-1 \mid k-1} & =\widehat{x}_{k-1 \mid k-1}+\rho U_{i} \sqrt{s_{i}} \\
i & =1,2, \cdots, n \\
x_{i, k-1 \mid k-1} & =\widehat{x}_{k-1 \mid k-1}-\rho U_{i} \sqrt{s_{i}} \\
i & =n+1, n+2, \cdots, 2 n
\end{aligned}\right.
$$

where $\rho$ is a scale coefficient that we can set equal to 1 . The symbols $s_{i}$ and $U_{i}$ are the $i$ th eigenvalue and eigenvector of $S_{k-1}$ and $U_{(k-1)}$. The weight of sigma points can be calculated by the formula:

$$
\left\{\begin{array}{l}
\omega_{m}^{0}=\frac{\lambda}{n+\lambda} \\
\omega_{c}^{0}=\frac{\lambda}{n+\lambda}+\left(1-\alpha^{2}+\beta\right) \\
\omega_{m}^{i}=\omega_{c}^{i}=\frac{1}{2(n+\lambda)}, i=1,2, \cdots, 2 n
\end{array}\right.
$$

where $\lambda$ denotes $\alpha^{2}(n+k)-n, \alpha$ and $k$ represent scaling and tuning parameters, respectively, $n$ is the dimension of the state vector $x$, and $\beta$ is a parameter related to the noise type.

(2) Transform the sigma sampling points using the nonlinear function $f(\cdot)$ :

$$
\left\{\begin{array}{l}
\phi_{i, k-1 \mid k-1}=f\left(x_{i, k-1 \mid k-1}, u_{k-1}\right), i=0,1, \ldots 2 n \\
D^{\eta} \widehat{x}_{k \mid k-1}=\sum_{i=0}^{2 n} \omega_{m}^{i} \phi_{i, k-1 \mid k-1} .
\end{array}\right.
$$

(3) Update the prior states estimation. The mean and covariance of $D^{\eta} x_{k}$ and $x_{k}$ can be calculated by:

$$
\begin{aligned}
& \left\{\begin{aligned}
P_{k \mid k-1}^{\Delta \Delta} & =\operatorname{Cov}\left[D^{\eta} x_{k \mid y_{k-1}}\right], \\
& =\sum_{i=0}^{2 n} \omega_{c}^{j}\left(\phi_{i, k-1 \mid k-1}-D^{\eta} \widehat{x}_{k \mid k-1}\right), \\
& \times\left(\phi_{i, k-1 \mid k-1}-D^{\eta} \widehat{x}_{k \mid k-1}\right)^{T}+Q \\
P_{k \mid k-1}^{\Delta \Delta} & =\operatorname{Cov}\left[x_{k-1}, D^{\eta} x_{k \mid y_{k-1}}\right], \\
& =\sum_{i=0}^{2 n} \omega_{c}^{j}\left(\phi_{i, k-1 \mid k-1}-D^{\eta} \widehat{x}_{k \mid k-1}\right), \\
& \times\left(\phi_{i, k-1 \mid k-1}-D^{\eta} \widehat{x}_{k \mid k-1}\right)^{T}, \\
\widehat{x}_{k \mid k-1} & =D^{\eta} \widehat{x}_{k \mid k-1}-\sum_{j=1}^{k}(-1)^{j} \gamma_{j} \widehat{x}_{k-j \mid k-j} \\
P_{k \mid k-1} & =P_{k \mid k-1}^{\Delta \Delta}+\gamma_{1} P_{k \mid k-1}^{x \Delta} \\
& +P_{k \mid k-1}^{\Delta x} \gamma_{1}+\sum_{j=1}^{k} \gamma_{j} P_{k-j \mid k-j} \gamma_{j} .
\end{aligned}\right.
\end{aligned}
$$




\section{Observation updating}

(1) Calculate sigma points using the singular value decomposition. The weight of the sigma points is obtained using (20):

$$
\left\{\begin{aligned}
P_{k \mid k-1} & =U_{k-1} S_{k-1} V_{k-1}^{T}, \\
x_{0, k-1 \mid k-1} & =\widehat{x}_{k-1 \mid k-1} \\
x_{i, k-1 \mid k-1} & =\widehat{x}_{k-1 \mid k-1}+\rho U_{i} \sqrt{s_{i}} \\
i & =1,2, \cdots, n \\
x_{i, k-1 \mid k-1} & =\widehat{x}_{k-1 \mid k-1}+\rho U_{i} \sqrt{s_{i}} \\
i & =n+1, n+2, \cdots, 2 n .
\end{aligned}\right.
$$

(2) Transform the sigma sampling points using the nonlinear function $h(\cdot)$ :

$$
\left\{\begin{array}{l}
\theta_{i, k \mid k-1}=h\left(x_{i, k \mid k-1}\right), i=0,1, \cdots, 2 n, \\
\widehat{y}_{k \mid k-1}=\sum_{i=0}^{2 n} \omega_{m}^{i} \theta_{i, k \mid k-1} .
\end{array}\right.
$$

(3) Estimate the observation-error covariance matrix:

$$
\left\{\begin{aligned}
P_{k \mid k-1}^{y y} & =\operatorname{Cov}\left[y_{k} \mid y_{k-1}\right], \\
& =\sum_{i=0}^{2 n} \omega_{c}^{j}\left(\theta_{i, k \mid k-1}-\widehat{y}_{k \mid k-1}\right), \\
& \times\left(\theta_{i, k \mid k-1}-\widehat{y}_{k \mid k-1}\right)^{T}+R \\
P_{k \mid k-1}^{x y} & =\operatorname{Cov}\left[x_{k}, y_{k} \mid y_{k-1}\right] \\
& =\sum_{i=0}^{2 n} \omega_{c}^{j}\left(\theta_{i, k \mid k-1}-\widehat{y}_{k \mid k-1}\right), \\
& \times\left(\theta_{i, k \mid k-1}-\widehat{y}_{k \mid k-1}\right)^{T}
\end{aligned}\right.
$$

(4) Calculate the theoretical and actual covariances:

$$
\left\{\begin{aligned}
M_{k} & =\sum_{i=0}^{2 n} \omega_{c}^{j}\left(\theta_{i, k \mid k-1}-\widehat{y}_{k \mid k-1}\right) \\
& \times\left(\theta_{i, k \mid k-1}-\widehat{y}_{k \mid k-1}\right)^{T}+R \\
N_{k} & =\frac{1}{n} \sum_{i}^{k}\left[y_{i}-y_{i \mid i-1}\right]\left[y_{i}-y_{i \mid i-1}\right]^{T} \\
i & =k-n+1
\end{aligned}\right.
$$

(5) Update the observation noise variance:

$$
\left\{\begin{array}{l}
G_{k}=M_{k}-N_{k} \\
\widehat{V_{k}}=\mu_{k} V_{k}
\end{array}\right.
$$

where $G_{k}$ is the input value of the fuzzy controller and $\mu_{k}$ is the output value as an adjusted factor through the fuzzy inference system. We can then obtain the new $P_{k \mid k-1}^{y y}$. 
(6) Update the posterior states estimation:

$$
\left\{\begin{aligned}
K_{k} & =P_{k \mid k-1}^{x y}\left(P_{k \mid k-1}^{y y}\right)^{-1}, \\
\widehat{x}_{k \mid k} & =\widehat{x}_{k \mid k-1}+K\left(y_{k}-\widehat{y}_{k \mid k-1}\right), \\
P_{k \mid k} & =P_{k \mid k-1}-K_{k} P_{k \mid k-1}^{y y} K_{k}^{T},
\end{aligned}\right.
$$

where $K_{k}$ is the Kalman filter gain. With the update of $V_{k}$, we can get the updated Kalman filter gain $K_{k}$ and the state error covariance matrix $P_{k \mid k}$.

\section{Numerical Verification and Discussion}

The current and voltage data under FUDS and BJDST conditions are used to verify the accuracy of the SOC estimation algorithm. The corresponding current and voltage profiles at the temperature of $25^{\circ} \mathrm{C}$ are shown in Figure 9. Due to limited space, we omit the current and voltage data at $0{ }^{\circ} \mathrm{C}$ and $45^{\circ} \mathrm{C}$, available at the CALCE Battery Research Group. To verify the validity and feasibility of the proposed method, we also compare the FFUKF with the EKF and FUKF algorithms.
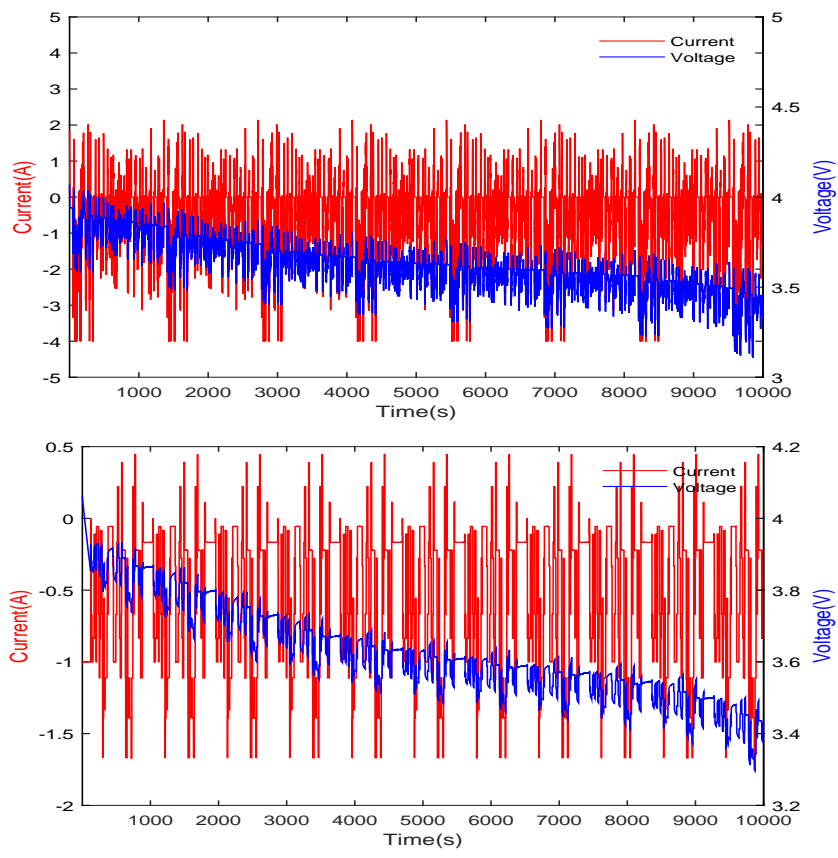

Figure 9. Current and voltage profiles of the operation conditions: FUDS and BJDST.

\subsection{Experimental Results at $25^{\circ} \mathrm{C}$}

Figures 10 and 11 show the SOC estimation results and estimation error, respectively. The blue line corresponds to the FFUKF. The red and magenta lines stand for FUKF and EKF, respectively. Also, the three algorithms are compared with the reference value represented by a black line. The closer to the reference, the higher the estimation accuracy of the algorithm. In order to see the differences between each algorithm more clearly, Figure 10 is partially magnified. We verify that the estimation results of the FFUKF are closer to the reference value. Under the two operating conditions, one can note that the FECM-based (FFUKF and FUKF) algorithm has higher accuracy than the ECM-based (EKF). Also, the FFUKF is more accurate than the FUKF. From Figure 11, the absolute estimation error of SOC of the FFUKF is no more than 0.005 under the two operating conditions, but the error of the other two algorithms is above 0.005 during the whole cycle. Even if disturbed by the noise environment, the FFUKF can still maintain high accuracy without large fluctuation, which shows that the proposed algorithm is stable to a certain extent. Additionally, it is clear that the error of the FFUKF is smoother than the one of the EKF, 
which confirms the superiority of the FFUKF in noisy environment. Table 3 summarizes the RMSE of the EKF, FUKF and FFUKF at $25^{\circ} \mathrm{C}$. Under the two operation conditions, the RMSE of the proposed algorithm is bellow $0.20 \%$. However, the RMSE of the other two algorithms are $0.68 \%$ and $1.95 \%$, respectively. This more clearly shows that our proposed algorithm has high accuracy over traditional methods.
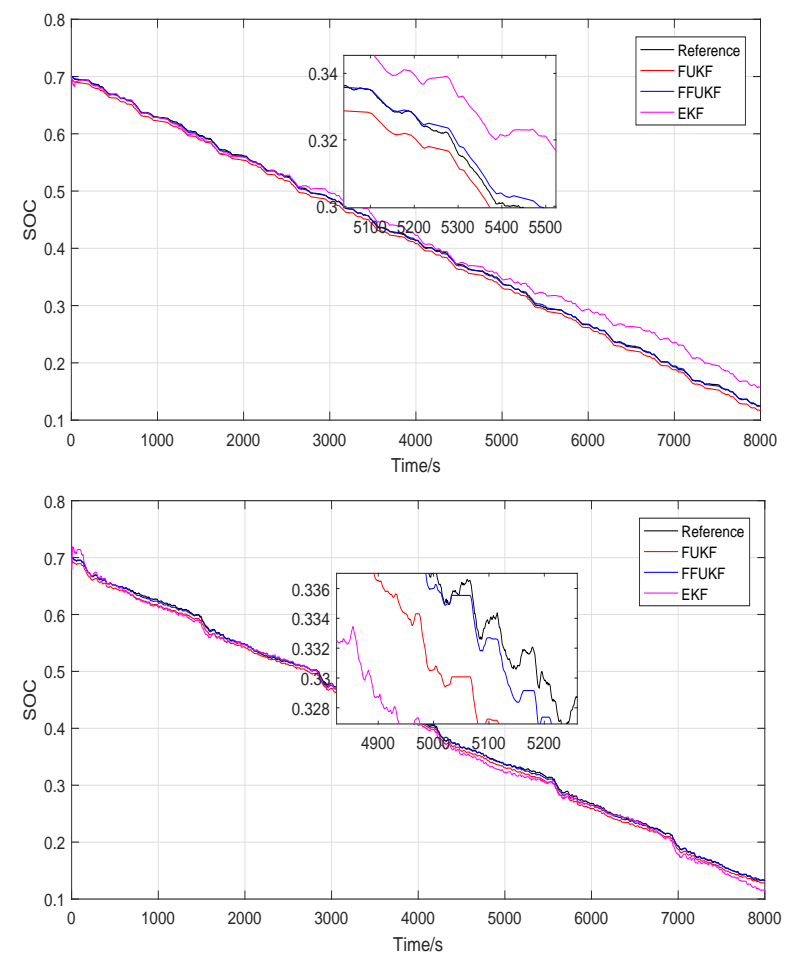

Figure 10. The SOC estimation curves under BJDST and FUDS at $25^{\circ} \mathrm{C}$.
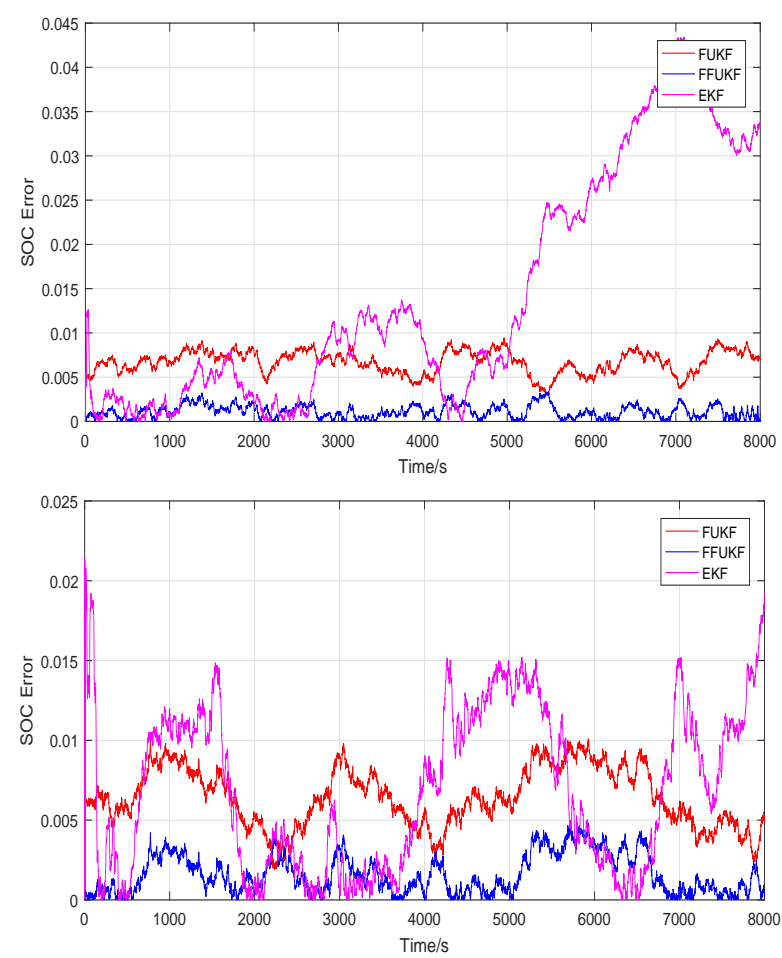

Figure 11. The SOC estimation error curves under BJDST and FUDS at $25^{\circ} \mathrm{C}$. 
Table 3. The RMSE under BJDST and FUDS at $25^{\circ} \mathrm{C}$.

\begin{tabular}{cccc}
\hline RMSE & EKF & FUKF & FFUKF \\
\hline FUDS & $0.87 \%$ & $0.67 \%$ & $0.20 \%$ \\
\hline BJDST & $1.95 \%$ & $0.68 \%$ & $0.13 \%$ \\
\hline
\end{tabular}

Because SOC estimation of lithium-ion batteries is affected by temperature, preserving the SOC estimation accuracy at different temperatures is crucial. As such, we carried out two sets of experiments at $0{ }^{\circ} \mathrm{C}$ and $45^{\circ} \mathrm{C}$ to demonstrate the robustness of the proposed method at different temperatures.

\subsection{Experimental Results at $0^{\circ} \mathrm{C}$}

Figures 12 and 13 show the SOC estimation results and the estimation error under two cases at $0{ }^{\circ} \mathrm{C}$, respectively. The ECM-based algorithm is obviously much worse than the FECM-based one in terms of accuracy. Figure 12 is partially magnified in order to highlight the differences. According to Figure 12, one can see that the blue line, representing the FFUKF, is closer to the reference value, which also shows that the estimation accuracy of the FFUKF is higher. From Figure 13, it is easy to see that the FFUKF can also maintain high accuracy at low temperatures. Most of the time, the estimation error of the FFUKF is kept within 0.005 . The estimation errors of the other two algorithms are more than 0.01 in most of the time. In addition, the estimation error of the EKF fluctuates greatly, which shows that the EKF is very unstable in low temperature environment. The FFUKF has small fluctuation, which shows that it can maintain good estimation accuracy and has a certain stability even at low temperature. Table 4 gives a more intuitive explanation through the RMSE. Under FUDS, the RMSE of the FFUKF is $0.20 \%$. However, the RMSE of the other two methods are more than $0.85 \%$. Meanwhile, the RMSE of the FFUKF is lower than that presented by traditional algorithm. This proves once again that our method is superior to the traditional methods in low temperature environment. Under the two conditions, although the result is worse than that under the temperature of $25^{\circ} \mathrm{C}$, the maximum error of the FFUKF is still less than $0.32 \%$.
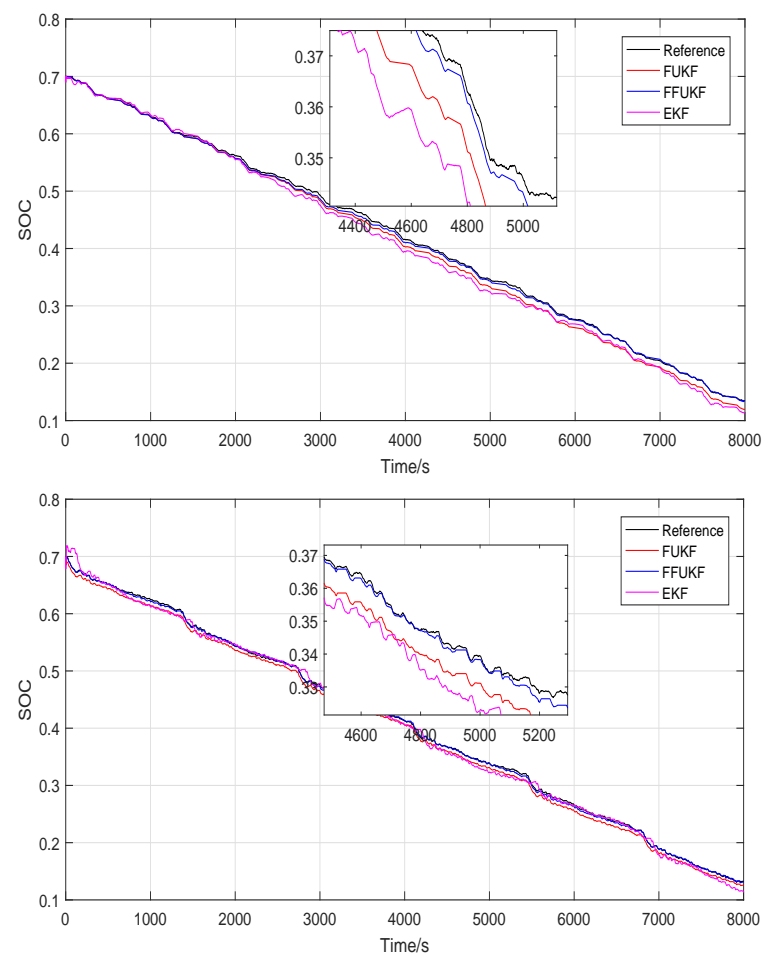

Figure 12. The SOC estimation curves under BJDST and FUDS at $0{ }^{\circ} \mathrm{C}$. 

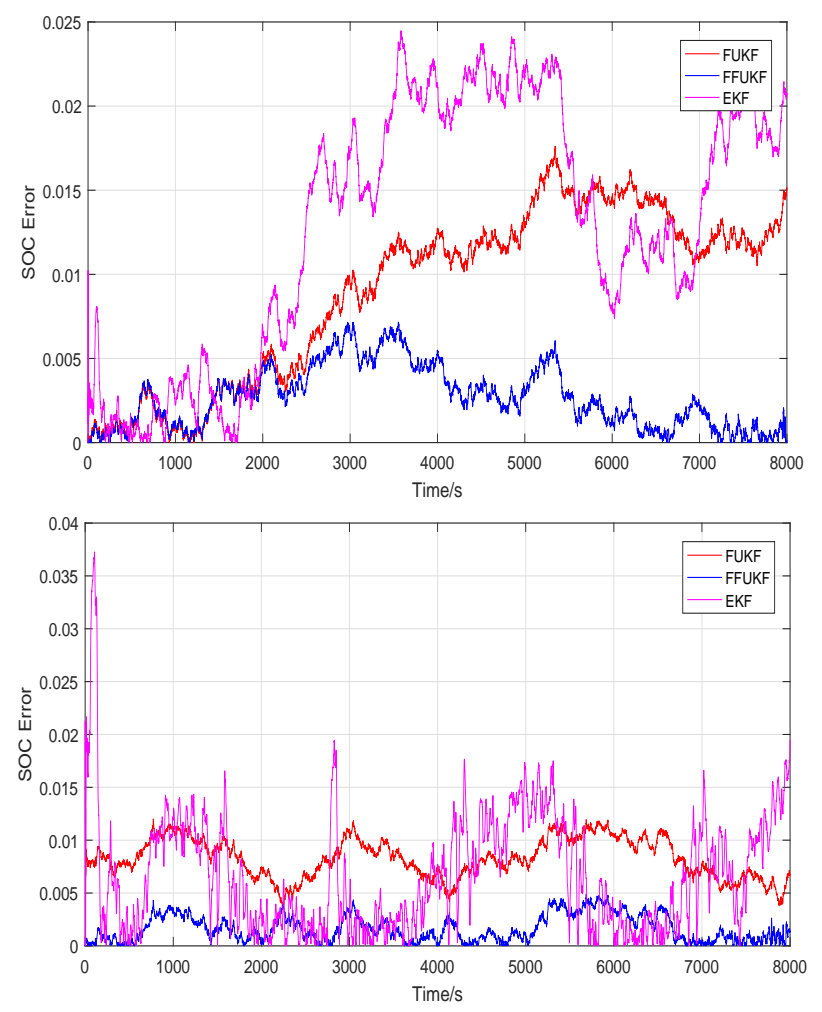

Figure 13. The SOC estimation error curves under BJDST and FUDS at $0{ }^{\circ} \mathrm{C}$.

Table 4. The RMSE under BJDST and FUDS at $0{ }^{\circ} \mathrm{C}$.

\begin{tabular}{cccc}
\hline RMSE & EKF & FUKF & FFUKF \\
\hline FUDS & $0.88 \%$ & $0.85 \%$ & $0.20 \%$ \\
\hline BJDST & $1.49 \%$ & $1.04 \%$ & $0.32 \%$ \\
\hline
\end{tabular}

\subsection{Experimental Results at $45^{\circ} \mathrm{C}$}

Figures 14 and 15 show the SOC estimation results and the estimation error under FUDS and BJDST at $45^{\circ} \mathrm{C}$, respectively. Figure 14 is also partially magnified so that we can more clearly observe which line is closer to the reference value represented by the black line. Undoubtedly, compared with the other two traditional algorithms, the FFUKF represented by the blue line is closer to the reference and has very high accuracy. Also, It follows from Figure 14, that the ECM-based algorithm (EKF) is much worse than the FECM-based (FUKF and FFUKF) one in terms of accuracy. At a higher temperature $\left(45^{\circ} \mathrm{C}\right)$, the FECM-based algorithm still reveals smaller error. From Figure 15, under the two working conditions, the SOC estimation error of our algorithm does not exceed 0.01 in most of the time, but the estimation error of the other two algorithms are much more than 0.01. Especially, in the case of FUDS, the error of the EKF varies quickly. The fluctuation of the FFUKF is smaller than that of the EKF. This further verifies that our algorithm based on the FFUKF has a certain stability at high temperature. The FFUKF still yields higher accuracy at a higher temperature. Table 5 gives a more intuitive explanation through RMSE. In both cases, the RMSE of the EKF and FUKF exceeds 1\%, but the RMSE of the FFUKF does not exceed $0.58 \%$. This not only shows that the FFUKF is superior to the other two traditional algorithms, but also maintains a certain accuracy under the condition of high temperature and noise. However, compared with low temperature $0{ }^{\circ} \mathrm{C}$ and normal temperature $25^{\circ} \mathrm{C}$, the accuracy is not good enough, which may be because the battery model we established is vulnerable to high temperature.

From the above three sets of experiments, one can conclude that the accuracy of the FFUKF is always better than the one obtained with the other two methods in all operating 
conditions (FUDS and BJDST). Moreover, although the operating conditions are poor, the RMSE can almost be kept within $0.58 \%$. It can maintain a certain stability even under the conditions of ambient temperature and noise. More important, the FFUKF solves the problem of low estimation accuracy caused by noise in practical operation. Obviously, the estimation accuracy of the algorithm is relatively higher at the same temperature.
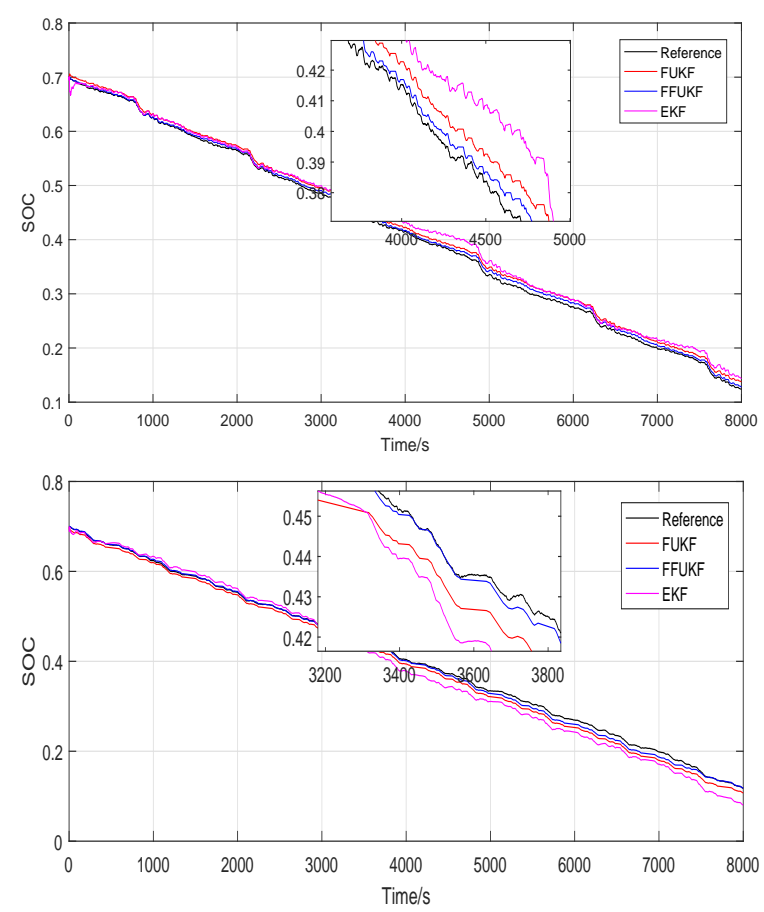

Figure 14. The SOC estimation curves under BJDST and FUDS at $45^{\circ} \mathrm{C}$.
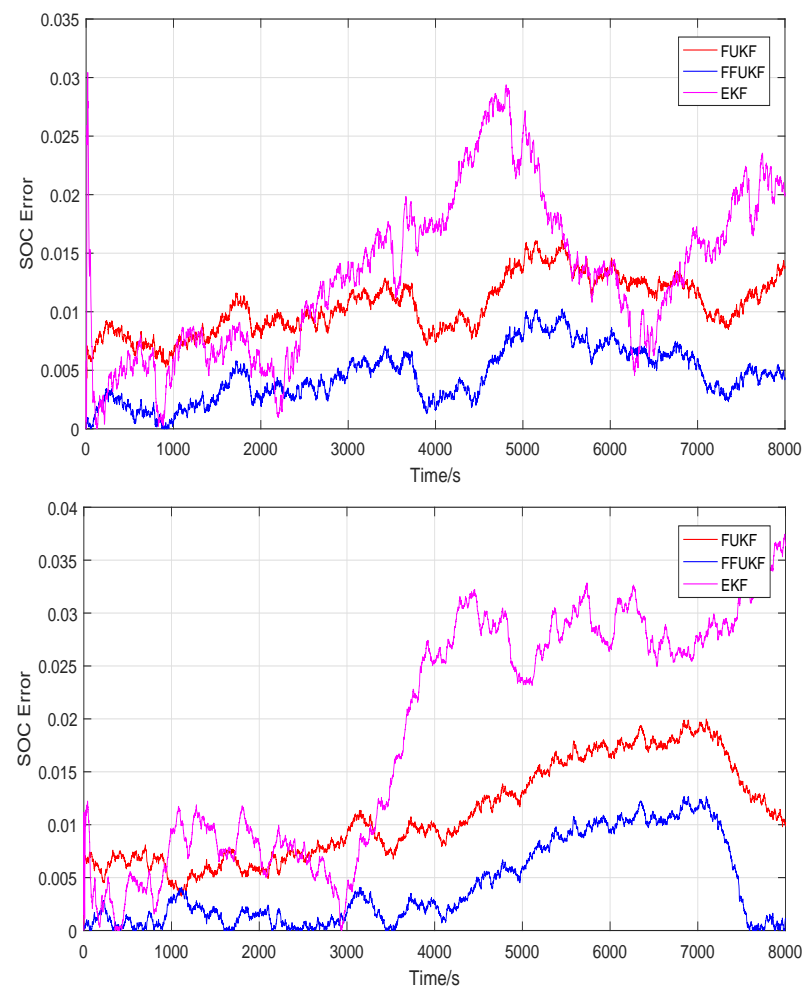

Figure 15. The SOC estimation error curves under BJDST and FUDS at $45^{\circ} \mathrm{C}$. 
Table 5. The RMSE under BJDST and FUDS at $45^{\circ} \mathrm{C}$.

\begin{tabular}{cccc}
\hline RMSE & EKF & FUKF & FFUKF \\
\hline FUDS & $1.49 \%$ & $1.08 \%$ & $0.51 \%$ \\
\hline BJDST & $2.18 \%$ & $1.20 \%$ & $0.58 \%$ \\
\hline
\end{tabular}

\section{Conclusions}

In this paper a new SOC estimation algorithm named fuzzy fractional-order unscented Kalman filter was proposed to estimate the SOC of lithium-ion batteries accurately. The method can infer the measurement noise in real time, so as to improve the influence of the measurement noise on the estimation results as the working conditions change. Compared with the EKF and FUKF algorithms, the experimental results indicated that the proposed method has better performance during the working conditions of BJDST and FUDS. It was also verified that the accuracy of the proposed algorithm is better than the EKF and FUKF at different temperatures.

Author Contributions: Methodology, L.C.; software, H.K.; validation and data curation, R.W.; writing — original draft preparation, Y.C.; writing—review and editing, A.M.L. and L.C. All authors have read and agreed to the published version of the manuscript.

Funding: This research was funded by the National Natural Science Funds of China (No. 62073114, No. 11971032) and Key Research and Development Project of Anhui Province (202104a05020035).

Institutional Review Board Statement: Not applicable.

Informed Consent Statement: Not applicable

Data Availability Statement: The data that support the findings of this study are available which has included references to the CALCE article that describes the experiments conducted for generating the data.

Conflicts of Interest: The authors declare no conflict of interest.

\section{References}

1. Fernández, R.Á. A more realistic approach to electric vehicle contribution to greenhouse gas emissions in the city. J. Clean. Prod. 2018, 172, 949-959. [CrossRef]

2. Larcher, D.; Tarascon, J.M. Towards greener and more sustainable batteries for electrical energy storage. Nat. Chem. 2015, 7, 19-29. [CrossRef] [PubMed]

3. Cheng, K.W.E.; Divakar, B.; Wu, H.; Ding, K.; Ho, H.F. Battery-management system (BMS) and SOC development for electrical vehicles. IEEE Trans. Veh. Technol. 2010, 60,76-88. [CrossRef]

4. Zhang, Q.; Li, Y.; Shang, Y.; Duan, B.; Cui, N.; Zhang, C. A fractional-order kinetic battery model of lithium-ion batteries considering a nonlinear capacity. Electronics 2019, 8, 394. [CrossRef]

5. He, H.; Xiong, R.; Guo, H.; Li, S. Comparison study on the battery models used for the energy management of batteries in electric vehicles. Energy Convers. Manag. 2012, 64, 113-121. [CrossRef]

6. Chen, Y.; Yang, G.; Liu, X.; He, Z. A time-efficient and accurate open circuit voltage estimation method for lithium-ion batteries. Energies 2019, 12, 1803. [CrossRef]

7. Aylor, J.H.; Thieme, A.; Johnso, B. A battery state-of-charge indicator for electric wheelchairs. IEEE Trans. Ind. Electron. 1992, 39, 398-409. [CrossRef]

8. Feng, F.; Lu, R.; Zhu, C. A combined state of charge estimation method for lithium-ion batteries used in a wide ambient temperature range. Energies 2014, 7, 3004-3032. [CrossRef]

9. Waag, W.; Fleischer, C.; Sauer, D.U. Critical review of the methods for monitoring of lithium-ion batteries in electric and hybrid vehicles. J. Power Sources 2014, 258, 321-339. [CrossRef]

10. Tian, J.; Xiong, R.; Shen, W.; Lu, J. State-of-charge estimation of LiFePO4 batteries in electric vehicles: A deep-learning enabled approach. Appl. Energy 2021, 291, 116812. [CrossRef]

11. Belhani, A.; M'Sirdi, N.K.; Naamane, A. Adaptive sliding mode observer for estimation of state of charge. Energy Procedia 2013, 377-386. [CrossRef]

12. Nath, A.; Gupta, R.; Mehta, R.; Bahga, S.S.; Gupta, A.; Bhasin, S. Attractive ellipsoid sliding mode observer design for state of charge estimation of lithium-ion cells. IEEE Trans. Veh. Technol. 2020, 69, 14701-14712. [CrossRef] 
13. Chen, Z.; Zhou, J.; Zhou, F.; Xu, S. State-of-charge estimation of lithium-ion batteries based on improved H infinity filter algorithm and its novel equalization method. J. Clean. Prod. 2021, 290, 125180. [CrossRef]

14. Li, L.; Hu, M.; Xu, Y.; Fu, C.; Jin, G.; Li, Z. State of charge estimation for lithium-ion power battery based on H-infinity filter Algorithm. Appl. Sci. 2020, 10, 6371. [CrossRef]

15. Tao, J.; Zhu, D.; Sun, C.; Chu, D.; Ma, Y.; Li, H.; Li, Y.; Xu, T. A novel method of SOC estimation for electric vehicle based on adaptive particle filter. Autom. Control. Comput. Sci. 2020, 54, 412-422.

16. Simon, D. Optimal State Estimation: Kalman, H Infinity, and Nonlinear Approaches; John Wiley \& Sons: Hoboken, NJ, USA, 2006.

17. Xiao, R.; Shen, J.; Li, X.; Yan, W.; Pan, E.; Chen, Z. Comparisons of modeling and state of charge estimation for lithium-ion battery based on fractional order and integral order methods. Energies 2016, 9, 184. [CrossRef]

18. Yang, S.; Zhou, S.; Hua, Y.; Zhou, X.; Liu, X.; Pan, Y.; Ling, H.; Wu, B. A parameter adaptive method for state of charge estimation of lithium-ion batteries with an improved extended Kalman filter. Sci. Rep. 2021, 11, 1-15.

19. Julier, S.J.; Uhlmann, J.K. Unscented filtering and nonlinear estimation. Proc. IEEE 2004, 92, 401-422. [CrossRef]

20. Zhang, S.; Guo, X.; Zhang, X. An improved adaptive unscented kalman filtering for state of charge online estimation of lithium-ion battery. J. Energy Storage 2020, 32, 101980. [CrossRef]

21. Han, J.; Kim, D.; Sunwoo, M. State-of-charge estimation of lead-acid batteries using an adaptive extended Kalman filter. J. Power Sources 2009, 188, 606-612. [CrossRef]

22. Sun, F.; Hu, X.; Zou, Y.; Li, S. Adaptive unscented Kalman filtering for state of charge estimation of a lithium-ion battery for electric vehicles. Energy 2011, 36, 3531-3540. [CrossRef]

23. Zhang, Z.; Jiang, L.; Zhang, L.; Huang, C. State-of-charge estimation of lithium-ion battery pack by using an adaptive extended Kalman filter for electric vehicles. J. Energy Storage 2021, 37, 102457. [CrossRef]

24. Zeng, M.; Zhang, P.; Yang, Y.; Xie, C.; Shi, Y. SOC and SOH joint estimation of the power batteries based on fuzzy unscented Kalman filtering algorithm. Energies 2019, 12, 3122. [CrossRef]

25. Lai, X.; Qiao, D.; Zheng, Y.; Zhou, L. A fuzzy state-of-charge estimation algorithm combining ampere-hour and an extended Kalman filter for Li-ion batteries based on multi-model global identification. Appl. Sci. 2018, 8, 2028. [CrossRef]

26. Victor, S.; Malti, R.; Garnier, H.; Oustaloup, A. Parameter and differentiation order estimation in fractional models. Automatica 2013, 49, 926-935. [CrossRef]

27. Chen, Y.; Huang, D.; Zhu, Q.; Liu, W.; Liu, C.; Xiong, N. A new state of charge estimation algorithm for lithium-ion batteries based on the fractional unscented Kalman filter. Energies 2017, 10, 1313. [CrossRef]

28. Xiong, R.; Tian, J.; Shen, W.; Sun, F. A novel fractional order model for state of charge estimation in lithium ion batteries. IEEE Trans. Veh. Technol. 2018, 68, 4130-4139. [CrossRef]

29. Sabatier, J.; Cugnet, M.; Laruelle, S.; Grugeon, S.; Sahut, B.; Oustaloup, A.; Tarascon, J. A fractional order model for lead-acid battery crankability estimation. Commun. Nonlinear Sci. Numer. Simul. 2010, 15, 1308-1317. [CrossRef]

30. Liu, C.; Liu, W.; Wang, L.; Hu, G.; Ma, L.; Ren, B. A new method of modeling and state of charge estimation of the battery. J. Power Sources 2016, 320, 1-12. [CrossRef]

31. Xu, J.; Mi, C.C.; Cao, B.; Cao, J. A new method to estimate the state of charge of lithium-ion batteries based on the battery impedance model. J. Power Sources 2013, 233, 277-284. [CrossRef]

32. Wei, Z.; Zou, C.; Leng, F.; Soong, B.H.; Tseng, K.J. Online model identification and state-of-charge estimate for lithium-ion battery with a recursive total least squares-based observer. IEEE Trans. Ind. Electron. 2017, 65, 1336-1346. [CrossRef]

33. Monje, C.A.; Chen, Y.; Vinagre, B.M.; Xue, D.; Feliu-Batlle, V. Fractional-Order Systems and Controls: Fundamentals and Applications; Springer Science \& Business Media: Berlin/Heidelberg, Germany, 2010.

34. Hu, X.; Li, S.; Peng, H. A comparative study of equivalent circuit models for Li-ion batteries. J. Power Sources 2012, 198, 359-367. [CrossRef]

35. Wang, B.; Liu, Z.; Li, S.E.; Moura, S.J.; Peng, H. State-of-Charge estimation for lithium-ion batteries based on a nonlinear fractional model. IEEE Trans. Control Syst. Technol. 2017, 25, 3-11. [CrossRef]

36. Hu, X.; Yuan, H.; Zou, C.; Li, Z.; Zhang, L. Co-estimation of state of charge and state of health for lithium-ion batteries based on fractional-order calculus. IEEE Trans. Veh. Technol. 2018, 67, 10319-10329. [CrossRef]

37. Aggab, T.; Avila, M.; Vrignat, P.; Kratz, F. Unifying model-based prognosis with learning-based time-series prediction methods: application to Li-Ion battery. IEEE Syst. J. 2021. [CrossRef]

38. Coronel-Escamilla, A.; Gómez-Aguilar, J.; Torres-Jiménez, J.; Mousa, A.; Elagan, S. Fractional synchronization involving fractional derivatives with nonsingular kernels: Application to chaotic systems. Math. Methods Appl. Sci. 2021. [CrossRef]

39. Zheng, F.; Xing, Y.; Jiang, J.; Sun, B.; Kim, J.; Pecht, M. Influence of different open circuit voltage tests on state of charge online estimation for lithium-ion batteries. Applied Energy 2016, 183, 513-525. [CrossRef]

40. Tavakoli, M.; Tabatabaei, M. Controllability and observability analysis of continuous-time multi-order fractional systems. Multidimens. Syst. Signal Process. 2017, 28, 427-450. [CrossRef] 